\title{
Pairing state with a time-reversal symmetry breaking in FeAs-based superconductors
}

\author{
Wei-Cheng Lee, ${ }^{1}$ Shou-Cheng Zhang, ${ }^{2}$ and Congjun $\mathrm{Wu}^{1}$ \\ ${ }^{1}$ Department of Physics, University of California, San Diego, CA 92093 \\ ${ }^{2}$ Department of Physics, McCullough Building, Stanford University, Stanford CA 94305-4045
}

\begin{abstract}
We investigate the competition between the extended $s_{ \pm}$-wave and $d_{x^{2}-y^{2}}$-wave pairing order parameters in the iron-based superconductors. Because of the frustrating pairing interactions among the electron and the hole fermi pockets, a time-reversal symmetry breaking $s+i d$ pairing state could be favored. We analyze this pairing state within the Ginzburg-Landau theory, and explore the experimental consequences. In such a state, spatial inhomogeneity induces supercurrent near a non-magnetic impurity and the corners of a square sample. The resonance mode between the $s_{ \pm}$ and $d_{x^{2}-y^{2}}$-wave order parameters can be detected through the $B_{1 g}$-Raman spectroscopy.
\end{abstract}

PACS numbers: 74.20. z, 71.10.Fd, 71.18. y, 71.20. b

The discovery of the iron-based new superconductors with high critical temperatures has attracted a great deal of attention [1, 2, 3, 4, 5, 6, 7]. The symmetry structure of Cooper pairs is one of the central issues for elucidating the superconducting properties. This problem is complicated by the structure of multiple Fermi surfaces in $\mathrm{LaO}_{1-x} \mathrm{~F}_{x} \mathrm{FeAs}$ systems, including hole Fermi surfaces $\alpha_{1,2}$ around the $\Gamma$-point at $\left(k_{x}, k_{y}\right)=(0,0)$, and the electron Fermi surfaces $\beta_{1,2}$ around the $\mathrm{M}_{1,2}$ points at $(\pi, 0)$ and $(0, \pi)$, respectively. Many theoretical proposals have suggested the fully-gapped extended $s_{ \pm^{-}}$ wave state which preserves the 4 -fold rotational symmetry [8, 9, 10, 11, 12, 13]. Experimentally, the superfluid density obtained from the penetration depth measurements is insensitive to temperature, consistent with this picture [14, 15, 16]. Another competing pairing structure in the square lattice is $d_{x^{2}-y^{2}}$ as proposed by several groups [9, 13, 17, 18]. Kuroki et al. 99] find that the $d_{x^{2}-y^{2}}$ pairing wins over the $s_{ \pm}$state if the pairing contribution from $\alpha$-Fermi surfaces is suppressed either by lowering the height of the As atom to the Fe plane or by heavily electron doping [19]. In particular, several theoretical studies have suggested that $s_{ \pm}$and $d_{x^{2}-y^{2}}$ pairings are nearly degnerate $19,20,21]$.

The calculation of the superconducting susceptibility in the five-band model shows that the extended $s$ wave and $d_{x^{2}-y^{2}}$ pairing orders compete with each other [9]. The spin susceptibility has peaked values around $\left(k_{x}, k_{y}\right)=(\pi, 0)$ and $(0, \pi)$ which corresponds to the nesting wavectors connecting $\alpha$ and $\beta$ Fermi surfaces, and also at nesting wavevectors around $(\pi, \pi / 2)$ and $(\pi / 2, \pi)$ connecting two $\beta$-surfaces. In the spin fluctuation mechanism, the pairing order parameters favor opposite signs on two Fermi surfaces connected by nesting wavevectors. The first nesting favors the extended $s$-wave pairing, with the opposite signs for the electron and the hole fermi

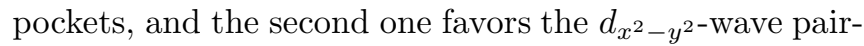
ing, with the opposite signs for the nearest-neighbor electron pockets, as depicted in Fig. 1. As one can see directly from Fig. [1, the pairing interactions based on these two different nesting vectors lead to a frustrating of the
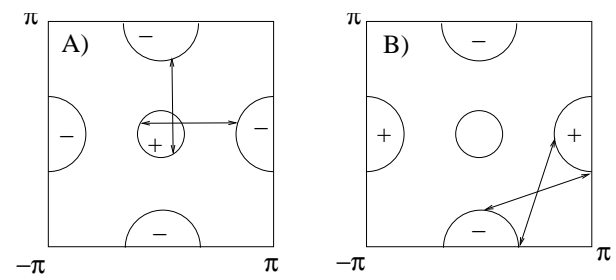

FIG. 1: The two competing pairing order parameters $\mathrm{LaFeAsO}_{1-x} \mathrm{~F}_{x}$ systems investigated in Ref. [8, 9] which are compatible with the square lattice geometry: A) the extended $s$-wave $\left(s_{ \pm}\right)$and $\left.\mathrm{B}\right)$ the $\mathrm{d}$-wave $\left(d_{x^{2}-y^{2}}\right)$. The nesting vectors are indicated by the bi-directional arrows.

pairing order parameters - the pure extended $s$ wave and the $d_{x^{2}-y^{2}}$ pairing states can not satisfy both nesting vectors simultaneously. In this situation, there arises a natural possibility of a mixed $s+i d$ pairing state, which can strike a compromise between the two nesting vectors.

In this article, we investigate the possibility of time-reversal (TR) symmetry breaking states in $\mathrm{LaO}_{1-x} \mathrm{~F}_{x} \mathrm{FeAs}$ systems based on the competition between the extended $s_{ \pm}$and $d_{x^{2}-y^{2}}$ order parameters. Based on a Ginzburg-Landau (GL) free energy analysis, we show that after the occurrence of the $s_{ \pm}$-pairing at $T_{c}$, the $s+i d$ pairing can develop at a lower temperature $T^{\prime}$ by breaking spatial rotation and TR symmetry under certain conditions. Spatial inhomogeneity can generate supercurrent around non-magnetic impurities and the corners of square samples due to a symmetry allowed quadratic gradient coupling of these two order parameters. The corner tunneling Josephson junction is analyzed. The resonance mode connecting two pairing order parameters can be measured through the $B_{1 g}$ Raman spectroscopy.

Unconventional superconductivity with TR symmetry breaking effect has been actively investigated in recent years. Much experimental evidence has shown that $\mathrm{Sr}_{2} \mathrm{RuO}_{4}$ exhibits TR symmetry breaking effects consistent with the $p_{x} \pm i p_{y}$ state, including the $\mu \mathrm{SR}$ and the Kerr effect [22, 23]. Recently, neutron scattering exper- 
iments indicate the existence of the loop current in the pseudogap region [24, 25, 26], and the Kerr effect has also been observed in the $\mathrm{YBa}_{2} \mathrm{Cu}_{3} \mathrm{O}_{6+x}$ system below the pseudogap temperature 27]. It has been pointed out that in multi-band superconductors TR symmetry breaking effect can exist with conventional pairing mechanism due to the repulsive interband Cooper pair scatterings [28, 29, 30, 31]. $\mathrm{LaO}_{1-x} \mathrm{~F}_{x} \mathrm{Fe}$ As systems naturally have multi-band structure, thus it would be interesting to investigate possible consequences of the TR symmetry breaking pairing states.

We construct a GL equation to describe the competition between these two singlet pair channels of the extended $s$-wave $\left(s_{ \pm}\right)$and $d$-wave $\left(d_{x^{2}-y^{2}}\right)$ order parameters as

$$
\begin{aligned}
F & =\alpha_{s}(T) \Delta_{s}^{*} \Delta_{s}+\alpha_{d}(T) \Delta_{d}^{*} \Delta_{d}+\beta_{1}\left|\Delta_{s}\right|^{4} \\
& +\beta_{2}\left|\Delta_{d}\right|^{4}+\beta_{3}\left|\Delta_{s}\right|^{2}\left|\Delta_{d}\right|^{2}+\beta_{4}\left\{\Delta_{s}^{*} \Delta_{s}^{*} \Delta_{d} \Delta_{d}+\text { c.c. }\right\} \\
& =\alpha_{s}(T) \Delta_{s}^{*} \Delta_{s}+\alpha_{d}(T) \Delta_{d}^{*} \Delta_{d}+\beta_{1}\left|\Delta_{s^{*}}\right|^{4}+\beta_{2}\left|\Delta_{d}\right|^{4} \\
& +\left(\beta_{3}+2 \beta_{4}\right)\left|\Delta_{s^{*}}\right|^{2}\left|\Delta_{d}\right|^{2}-\beta_{4} L^{2},
\end{aligned}
$$

where $\alpha_{s} \approx N_{0} \ln \left(T / T_{s}\right)$ and $\alpha_{d} \approx N_{0} \ln \left(T / T_{d}\right) ; N_{0}$ is the density of states at the Fermi energy; $\beta$ s are not sensitive to temperatures; $L=i\left(\Delta_{s}^{*} \Delta_{d}-\Delta_{d}^{*} \Delta_{s}\right)$. Because $\Delta_{s}$ and $\Delta_{d}$ belong to different representations of the lattice symmetry group, they do not couple at the quadratic level but can couple at the quartic level. In particular, the $\beta_{4}$-term is allowed by symmetry because orbital angular momentum is conserved modulo 4 . The thermodynamic stability condition requires that $\beta_{1,2}>0$ and $4 \beta_{1} \beta_{2}-\left(\beta_{3}-2\left|\beta_{4}\right|\right)^{2}>0$. $\beta_{3}$ is also expected to be positive because of the competing nature between $\Delta_{s}$ and $\Delta_{d}$. A similiar GL theory has been discussed in the high- $T_{c}$ cuprates 32 .

We assume that the pairing tendency in the $s_{ \pm}$is stronger but very close to that in the $d_{x^{2}-y^{2}}$ channel, i.e., $T_{s}>T_{d}$ and $1-T_{d} / T_{s} \ll 1$. By minimizing the GL free energy of Eq. 1, the condition for the appearance of $\Delta_{d}$ at temperature $T$ is: $-\alpha_{d}(T)>-\alpha_{s}(T) \lambda$, where $\lambda=\left(\beta_{3}-2\left|\beta_{4}\right|\right) /\left(2 \beta_{1}\right)$. This condition can be satisfied at $\lambda<1$ which gives rise to another critical temperature $T^{\prime}$ as: $T^{\prime}=\sqrt{T_{d} T_{s}}\left(T_{d} / T_{s}\right)^{\frac{1}{2} \frac{1+\lambda}{1-\lambda}}<T_{d}$ below which $\Delta_{d}$ develops. The sign of $\beta_{4}$ determines whether TR symmetry is broken or not. If $\beta_{4}>0$, it favors a phase difference of $\pm \frac{\pi}{2}$ between $\Delta_{s}$ and $\Delta_{d}$, i.e., the $s+i d$ pairing. On the other hand, the real combination of $s+d$ is realized at $\beta_{4}<0$, which preserves TR symmetry. In both cases, the 4 -fold rotational symmetry is broken at $T<T^{\prime}$ which corresponds to an Ising transition. In the former case a combined rotation of $90^{\circ}$ and TR operation still leave the system invariant.

While the uniform components of the $\Delta_{s}$ and the $\Delta_{d}$ components do not couple at the quadratic level, their gradient terms can. The general gradient terms of the

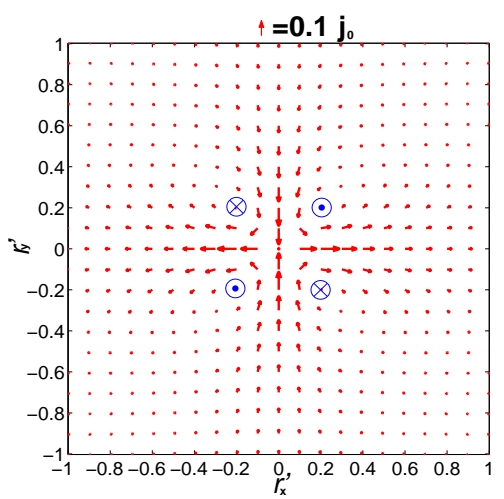

FIG. 2: Supercurrent induced by the impurity at $\vec{r}=(0,0)$ for $\beta_{4}>0$. We introduce the length scale $\xi=\sqrt{\gamma_{s} /\left|\alpha_{s}\right|}$, energy scale $\Delta_{0}=\sqrt{\left|\alpha_{s}(T)\right| / 2 \beta_{1}}$, and the density of states $N_{0}$. The dimensionless parameters are defined as $r_{i}^{\prime}=r_{i} / \xi$, $\alpha_{i}^{\prime} \equiv \alpha_{i} / N_{0}, \beta_{i}^{\prime} \equiv \beta_{i} \Delta_{0}^{2} / N_{0}, \gamma_{i}^{\prime} \equiv \gamma_{i} / \xi^{2} N_{0}$, with the values used here given by $r_{0}^{\prime}=0.1, \alpha_{0}^{\prime}=10, \alpha_{s}^{\prime}(T)=-1.0, \alpha_{d}^{\prime}(T)=$ $-0.75, \beta_{1}^{\prime}=1.0, \beta_{2}^{\prime}=1.0, \beta_{3}^{\prime}=0.5, \beta_{4}^{\prime}=0.4, \gamma_{s}^{\prime}=1.0, \gamma_{d}^{\prime}=$ 0.5 , and $\gamma_{s d}^{\prime}=0.35$. The supercurrent is plotted in unit of $j_{0}=e \alpha_{s}^{2}(T) \xi / \beta_{1}$ and the length of each arrow is proportional to the magnitude of the supercurrent. $\odot$ and $\otimes$ indicate the vorticities of the loop currents appearing in the four quadrants near the impurity.

\section{GL free energy are given by}

$$
\begin{aligned}
F_{\text {grad }} & =\gamma_{s}\left|\vec{\Pi} \Delta_{s}\right|^{2}+\gamma_{d}\left|\vec{\Pi} \Delta_{d}\right|^{2}+\gamma_{s d}\left(\Pi_{x}^{*} \Delta_{s}^{*} \Pi_{x} \Delta_{d}\right. \\
& \left.-\Pi_{y}^{*} \Delta_{s}^{*} \Pi_{y} \Delta_{d}+\text { c.c. }\right),
\end{aligned}
$$

where $\vec{\Pi}=\vec{\nabla}-2 i e \vec{A}$ and $A$ is the magnetic vector potential; the $\gamma_{s d}$-term describes the coupling between the $\Delta_{s, d}$ orders allowed by the square lattice structure [33]. Minimizing the free energy we arrive the coupled equations of

$$
\begin{aligned}
& \alpha_{s} \Delta_{s}+2 \beta_{1}\left|\Delta_{s}\right|^{2} \Delta_{s}+\beta_{3}\left|\Delta_{d}\right|^{2} \Delta_{s}+2 \beta_{4} \Delta_{d}^{2} \Delta_{s}^{*} \\
- & \gamma_{s}\left(\Pi_{x}^{2}+\Pi_{y}^{2}\right) \Delta_{s}-\gamma_{s d}\left(\Pi_{x}^{2}-\Pi_{y}^{2}\right) \Delta_{d}=0, \\
& \alpha_{d} \Delta_{d}+2 \beta_{2}\left|\Delta_{d}\right|^{2} \Delta_{d}+\beta_{3}\left|\Delta_{s}\right|^{2} \Delta_{d}+2 \beta_{4} \Delta_{s}^{2} \Delta_{d}^{*} \\
- & \gamma_{d}\left(\Pi_{x}^{2}+\Pi_{y}^{2}\right) \Delta_{d}-\gamma_{s d}\left(\Pi_{x}^{2}-\Pi_{y}^{2}\right) \Delta_{s}=0 .
\end{aligned}
$$

The electric supercurrent can be represented as

$$
\begin{aligned}
\vec{j} & =-\frac{\delta F}{\delta \vec{A}}=\vec{j}_{s}+\vec{j}_{d}+\vec{j}_{d s} \\
\vec{j}_{s} & =2 i e \gamma_{s}\left[\Delta_{s}^{*} \vec{\Pi} \Delta_{s}\right]+c . c . \\
\vec{j}_{d} & =2 i e \gamma_{d}\left[\Delta_{d}^{*} \vec{\Pi} \Delta_{d}\right]+c . c . \\
\vec{j}_{d s} & =2 i e \gamma_{s d}\left\{\left(\Delta_{s}^{*} \Pi_{x} \Delta_{d}+\Delta_{d}^{*} \Pi_{x} \Delta_{s}\right) \hat{e}_{x}-\left(\Delta_{s}^{*} \Pi_{y} \Delta_{d}\right.\right. \\
& \left.\left.+\Delta_{d}^{*} \Pi_{y} \Delta_{s}\right) \hat{e}_{y}\right\}+c . c .
\end{aligned}
$$

where $\vec{j}_{s, d}$ are the intra-component supercurrent, and $j_{s d}$ is the inter-component supercurrent. The total supercurrent $\vec{j}$ satisfies the continuity condition: $\vec{\nabla} \cdot \vec{j}=0$. 


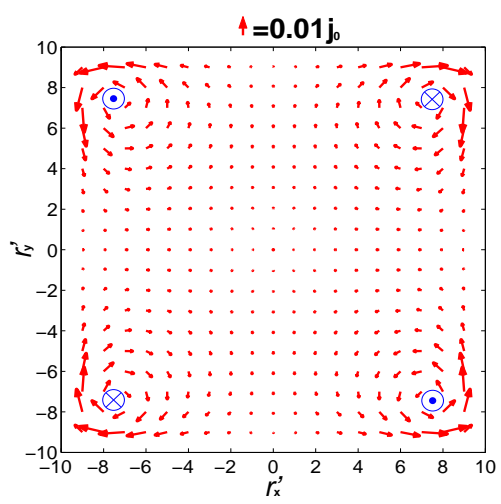

FIG. 3: Supercurrent distribution of a square sample in terms of the unit of $j_{0}$ with the same parameters as in Fig. 2 Current loops develop with the positive chirality at the rightdown and left-up corners and the negative chirality at the other two corners.

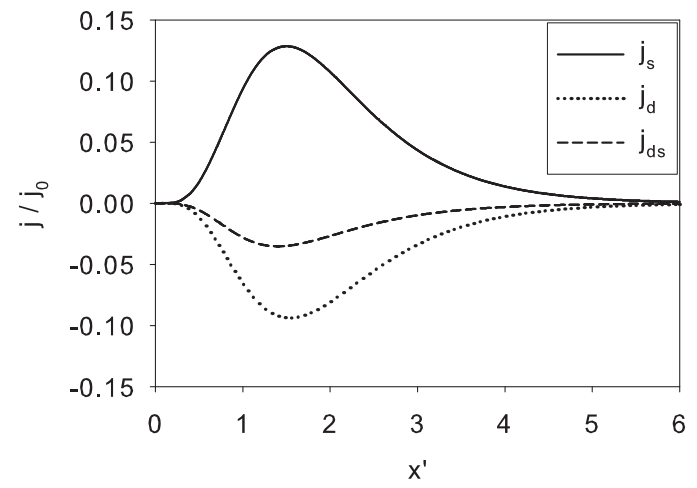

FIG. 4: Supercurrent components $\vec{j}_{s}, \vec{j}_{d}$ and $\vec{j}_{s d}$ in a sample of half plane geometry $(x>0)$ plotted with the unit of $j_{0}$. The total current $\vec{j}=\vec{j}_{s}+\vec{j}_{d}+\vec{j}_{s d}$ vanishes. The parameters are the same as in Fig 2

A novel consequence of the $\gamma_{s d}$-term is that spatial inhomogeneity induces supercurrent in a $s+i d$ superconductor. We consider a non-magnetic impurity located at $\vec{r}_{0}=(0,0)$ modeled as a Gaussian distribution of $\alpha \mathrm{s}$ $\alpha_{s}(\vec{r}, T)=\alpha_{s, d}(T)+\alpha_{0} e^{-r^{2} / r_{0}^{2}}$ with $\alpha_{0}>0$. At $T<T^{\prime}$, a $s+i d$ superconductor has the relative phase $\theta_{s d}= \pm \frac{\pi}{2}$ between $\Delta_{s, d}$ in the homogeneous system. However, in the presence of impurities, the spatial inhomogeneous distribution of order parameters causes the inhomogeneity of the relative phase $\theta_{s d}$ due to the $\gamma_{s d}$ term, which induces supercurrent near the impurity. We solve Eqs. 3 and plot the supercurrent pattern in Fig. 2. Assuming the magnetic field generated by the impurity-induced supercurrent is small, we neglect the dependence of $\vec{j}$ on the magnetic vector potential $\vec{A}$. The magnitude of the supercurrent rapidly decays beyond the order of the healing length defined $\xi=\sqrt{\frac{\gamma_{s}}{\left|\alpha_{s}\right|}}$. The suppercurrent pattern is symmetric under the rotation of $180^{\circ}$, and a combined operation of TR and the rotation of $\pm 90^{\circ}$. Along the $x$ and $y$-axis passing the impurity, the supercurrents flow along these axis and exhibit the pattern of "two in" and "two out", which is consistent with the reflection symmetry respect to the $x$ and $y$-axis and the continuity condition. In addition, the system has the combined TR and reflection symmetry respect to the diagonal axes of $\pm 45^{\circ}$, thus supercurrents flow perpendicularly to these axes. This results in staggered current loops and thus small staggered fluxes in the four quadrants near the impurity. However, these current loops arise from both amplitude and phase variations and do not possess quantized fluxes, and thus are not of topological nature. At $T_{c}>T>T^{\prime}$, a similar effect also occurs since a non-zero $d$-wave order parameter can be induced despite the supercurrent in this temperature range is very small. On the other hand, if a $s+d$ mixing is realized below $T^{\prime}$ at $\beta_{4}<0$, supercurrents will not be induced by spatial inhomogeneity because $s+d$ does not breaking TR symmetry. The spatial inhomogeneity only causes the amplitude variation of $\Delta_{s, d}$, whose relative phase difference remains fixed at $\theta_{s d}=0$ or $\pi$.

We next consider a square sample and investigate the supercurrent at the boundary. The spatial variation of $\Delta_{s, d}$ is along directions normal to edges. The continuity condition suppresses the supercurrent except at the four corners as depicted in Fig. 3. Each of four corners develop a circulating supercurrent loop whose chirality are staggered as we move around the edges. This is also consistent with the combined symmetry operation of TR and the rotation of $90^{\circ}$. Because at each corner the supercurrent has the same chirality, thus is easier to be detected by using SQUID than the single impurity case.

Furthermore, we consider the spatial distribution of $\Delta_{s, d}$ at $\beta_{4}>0$ along the edge of a half-plane sample with $x>0$ and the boundary of the $y$-axis. Given the boundary conditions of $\Delta_{s}(x=0, y)=\Delta_{d}(x=0, y)=0$ and the spatial homogeneity along the $y$-direction, Eq. 3 reduces to coupled 1D equations. Again the continuity condition forbids the appearances of a net supercurrent for a $s+i d$ superconductor, although the three components of the supercurrent, $j_{s}, j_{d}$, and $j_{s d}$, are nonzero individually as depicted in Fig. 4. One important feature is that $j_{s}$ and $j_{d}$ have opposite signs. This internal counterflow supercurrents is a result of the $\gamma_{s d}$ term. By plugging $\Delta_{s, d}(x)=\rho_{s, d}(x) e^{i \theta_{s, d}(x)}$ into the GL free energy, such a term can be approximated as: $2 \gamma_{s d} \sin \left(\theta_{s}-\theta_{d}\right)\left[\left(\partial_{x} \theta_{s}\right)\left(\partial_{x} \rho_{d}\right) \rho_{s}-\left(\partial_{x} \theta_{d}\right)\left(\partial_{x} \rho_{s}\right) \rho_{d}\right]$, and therefore the free energy can be lowered by choosing $\operatorname{sgn}\left(\partial_{x} \theta_{s}\right)=-\operatorname{sgn}\left(\partial_{x} \theta_{d}\right)$.

The Josephson junctions touching edges with different orientations of a single crystal sample act as convincing phase sensitive measurements for the $d$-wave pairing symmetry in high $\mathrm{T}_{c}$ systems 34, 35] and $p$-wave pairing symmetry in $\mathrm{Sr}_{2} \mathrm{RuO}_{4}$ [36, 37]. We consider the same 
conner junction in which the two adjacent faces of a single crystal FeAs superconductor are connected via Josephson weak coupling with a conventional $s$-wave superconducting thin film. We define that $J_{s, d}$ are the Josephson coupling between the $s_{ \pm}(d)$ order parameter and the conventional $s$-wave order parameter, respectively. Assuming that the two junctions at two adjacent faces are equal, we express the critical current with applied magnetic flux $\Phi_{\text {ext }}$ as [38]: $I_{c}\left(\Phi_{\text {ext }}\right)=2 I_{0} \cos \left[\pi\left(\Phi_{\text {ext }} / \Phi_{0}\right)+\delta / 2\right]$, where $\delta=\tan ^{-1}\left[2 e_{J} \sin \theta_{s d} /\left(1-e_{J}^{2}\right)\right], e_{J} \equiv J_{d} / J_{s}$, and $\Phi_{0}$ is the flux quantum. $\delta$ could only be 0 or $\pi$ for the $s+d$ mixing depending on which component is dominate, while it can be any value between 0 and $\pi$ for the case of $s+i d$ mixing.

Now we consider the case of $\left\langle\hat{\Delta}_{s}\right\rangle=\Delta_{0}$ and $\left\langle\hat{\Delta}_{d}\right\rangle=$ 0 . In this case we predict that a new collective mode can be observed in the $B_{1 g}$-mode of Raman spectroscopy, which behaves as the resonance mode connecting $\hat{\Delta}_{s, d}$, and is approximately the nematic operator $\hat{N}_{d}$ associated with the $B_{1 g}$ mode in the Raman spectroscopy. One can check the following commutator: $\left[\hat{N}_{d}, \hat{\Delta}_{d}^{\dagger}\right]=-2 \hat{\Delta}_{s}^{\dagger}+\hat{\Delta}_{s}^{\dagger \prime}$, where $\hat{N}_{d}=$ $1 / V \sum_{k}\left(\cos k_{x}-\cos k_{y}\right) c_{\vec{k} \sigma}^{\dagger} c_{\vec{k} \sigma}, \hat{\Delta}_{d}^{\dagger}=1 / V \sum_{\vec{k}}\left(\cos k_{x}-\right.$ $\left.\cos k_{y}\right) c_{\vec{k} \uparrow}^{\dagger} c_{-\vec{k} \downarrow}^{\dagger}, \hat{\Delta}_{s}^{\dagger}=1 / V \sum_{\vec{k}} \cos k_{x} \cos k_{y} c_{\vec{k} \uparrow}^{\dagger} c_{-\vec{k} \downarrow}^{\dagger}$, and $\Delta_{s}^{\dagger, \prime}=1 / V \sum_{\vec{k}}\left(\cos ^{2} k_{x}+\cos ^{2} k_{y}\right) c_{\vec{k} \uparrow}^{\dagger} c_{-\vec{k} \downarrow}^{\dagger}$. Since $\left\langle\Delta_{s}\right\rangle \neq$ $0, \Delta_{d}$ and $N_{d}$ are conjugate variables which lead to collective modes similiar to the $\pi$ resonance mode in cuprates [39, 40]: $\omega_{\text {res }} \sim \frac{\sqrt{K^{N_{d} K^{\Delta_{d}}}}}{C}$, where $K^{N_{d}} \sim N_{0} \Delta_{0}^{2}$ and $K^{\Delta_{d}} \sim \alpha_{d}^{*}(T)=\alpha_{d}+\left(\beta_{3}-2\left|\beta_{4}\right|\right) \Delta_{0}^{2}$ are the stiffnesses for $N_{d}$ and $\Delta_{d}$ respectively, and $C \sim N_{0}$ is the Berry curvature between $N_{d}$ and $\Delta_{d}$. Following the same arguement in Ref. 39, 41], the excited state with energy $\omega^{\text {res }}$ can be defined as: $\left|\Delta_{d}\right\rangle=\hat{\Delta}_{d}^{\dagger}|0\rangle$, where $|0\rangle$ is the BCS ground state. As a result, at $T=0$ the Raman response function of the $B_{1 g}$ mode becomes:

$$
\begin{gathered}
\chi^{B_{1 g}}(\omega)=\sum_{n}\left\{\frac{\left|\left\langle 0\left|N_{d}\right| n\right\rangle\right|^{2}}{\omega-\omega_{n}+i \epsilon}-\frac{\left|\left\langle 0\left|N_{d}\right| n\right\rangle\right|^{2}}{\omega+\omega_{n}-i \epsilon}\right\} \\
\approx\left\{\frac{\left|\left\langle 0\left|\left[N_{d}, \hat{\Delta}_{d}^{\dagger}\right]\right| 0\right\rangle\right|^{2}}{\omega-\omega^{r e s}+i \epsilon}-\frac{\left|\left\langle 0\left|\left[N_{d}, \hat{\Delta}_{d}^{\dagger}\right]\right| 0\right\rangle\right|^{2}}{\omega+\omega^{r e s}-i \epsilon}\right\} \\
\sim\left|\Delta_{s}\right|^{2}\left\{\frac{1}{\omega-\omega^{r e s}+i \epsilon}-\frac{1}{\omega+\omega^{r e s}-i \epsilon}\right\},
\end{gathered}
$$

which has a sharp peak at $\omega=\omega^{\text {res }}$. The temperature dependence of $\omega^{\text {res }}$ may be complex. Nevertheless, the revelence of $\Delta_{d}$ can still be infered from it. If the $\Delta_{d}$ is competing to $\Delta_{s}$ but the mixed state is not favored $(\lambda>1), \omega^{r e s}$ should have weak temperature dependence and remain finite as $T \rightarrow 0$. On the other hand, if the mixed state could occur at $T=T^{\prime}(\lambda<1)$, $\omega^{\text {res }}$ should appear after $T<T_{c}$ and decrease dramatically to zero as $T$ is approaching $T^{\prime}$.

In conclusion, we have investigated the competition between the extended $s$-wave and $d$-wave Cooper pairing orders in the FeAs-based superconductors. The multiple nesting wavevectors naturally leads to the possibility of a $s+i d$ pairing state which breaks the TR symmetry. Based on a general Ginzburg-Landau theory we have shown that in such a state supercurrent can be induced by spatial inhomogeneity, and several possible experiments to detect this state are discussed. We also proposed that a new collective mode should be observed in the $B_{1 g}$ Raman spectroscopy as the resonant mode between the two competing order parameters.

We thank X. Dai, Z. Fang, J. Hirsch, and J. Hu for helpful discussions. C. W. thanks the Aspen institute of Physics where part of the work was done. S. C. Z. is supported by the NSF DMR-0342832 and the US DOE under Contract No. DE-AC03-76SF00515. C. W. is supported by the startup fund and the Academic Senate research grant at UCSD, the Sloan Research Foundation, and ARO-W911NF0810291.

[1] Y. Kamihara et al., J. Am. Chem. Soc. 130, 3296 (2008).

[2] X. H. Chen et al., Nature 453, 761 (2008).

[3] G. F. Chen et al., Phys. Rev. Lett. 100, 247002 (2008).

[4] H. H. Wen et al., Europhys. Lett. 82, 17009 (2008).

[5] Z. A. Ren et al., Europhys. Lett. 83, 17002 (2008).

[6] M. Rotter et al., Phys. Rev. B 78, 020503 (2008).

[7] C. Wang et al., Europhys. Lett. 83, 67006 (2008).

[8] I. Mazin et al., Phys. Rev. Lett. 101, 057003 (2008).

[9] K. Kuroki et al., Phys. Rev. Lett. 101, 087004 (2008);

[10] D. Parker et al., Phys. Rev. B 78, 134524 (2008).

[11] A. V. Chubukov et al., Phys. Rev. B 78, 134512 (2008).

[12] Y. Bang and H.-Y. Choi, Phys. Rev. B 79, 054529 (2009).

[13] K. Seo et al, Phys. Rev. Lett. 101, 206404 (2008).

[14] H. Luetkens et al., Phys. Rev. Lett. 101, 097009 (2008).

[15] L. Malone et al., Phys. Rev. B 79, 140501 (2009).

[16] K. Hashimoto et al., Phys. Rev. Lett. 102, 017002 (2009).

[17] W. Q. Chen et-al., Phys. Rev. Lett. 102, 047006 (2009).

[18] X.-L. Qi et al., arXiv.org:0804.4332, (2008).

[19] K. Kuroki et al., arXiv.org:0904.261, (2009).

[20] S. Graser et al., New J. Phys. 11, 025016 (2009).

[21] H. Zhai et al., arXiv.org:0905.1171 (2009).

[22] G. M. Luke et al., Nature 394, 558 (1998).

[23] J. Xia et al., Phys. Rev. Lett. 97, 167002 (2006).

[24] B. Fauqué et al., Phys. Rev. Lett. 96, 197001 (2006).

[25] C. M. Varma, Phys. Rev. B 73, 155113 (2006).

[26] V. Aji et al., Phys. Rev. Lett. 99, 067003 (2007).

[27] J. Xia et al., Phys. Rev. Lett. 100, 127002 (2008).

[28] D. F. Agterberg et al., Phys. Rev. B 60, 14868 (1999).

[29] Y. Tanaka, J. Phys. Soc. Jpn. 70, 2844 (2001).

[30] D. F. Agterberg et al., Phys. Rev. B 66, 214507 (2002).

[31] T.-K. Ng and N. Nagaosa, arXiv.org:0809.3343, (2008).

[32] G. Kotliar, Phys. Rev. B 37, 3664 (1988).

[33] Y. Ren et al., Phys. Rev. Lett. 74, 3680 (1995).

[34] C. C. Tsuei et al., Rev. Mod. Phys. 72, 969 (2000).

[35] D. J. Van Harlingen, Rev. Mod. Phys. 67, 515 (1995).

[36] F. Kidwingira et al., Science 314, 1267 (2006).

[37] K. D. Nelson et al., Science 306, 1151 (2004).

[38] J. Annett et al., arXiv.org cond-mat/9601060, (1996).

[39] E. Demler et al., Rev. Mod. Phys. 76, 909 (2004). 
[40] W.-C. Lee et al., Phys. Rev. B 77, 214518 (2008).

[41] S. C. Zhang, Science 275, 1089 (1997). 